\title{
Alcohol and tobacco consumption affects bacterial richness in oral cavity mucosa biofilms
}

\author{
Andrew Maltez Thomas ${ }^{1,2}$, Frederico Omar Gleber-Netto ${ }^{1,2}$, Gustavo Ribeiro Fernandes ${ }^{1}$, Maria Amorim ${ }^{1,2}$, \\ Luisa Fernanda Barbosa ${ }^{1,3}$, Ana Lúcia Noronha Francisco ${ }^{4}$, Arthur Guerra de Andrade ${ }^{5}$, João Carlos Setubal ${ }^{6,7}$, \\ Luiz Paulo Kowalski ${ }^{4}$, Diana Noronha Nunes ${ }^{1}$ and Emmanuel Dias-Neto ${ }^{1,8^{*}}$
}

\begin{abstract}
Background: Today there are more than 2 billion alcohol users and about 1.3 billion tobacco users worldwide. The chronic and heavy use of these two substances is at the heart of numerous diseases and may wreak havoc on the human oral microbiome. This study delves into the changes that alcohol and tobacco may cause on biofilms of the human oral microbiome. To do so, we used swabs to sample the oral biofilm of 22 subjects; including 9 controlindividuals with no or very low consumption of alcohol and no consumption of tobacco, 7 who were chronic and heavy users of both substances and 6 active smokers that reported no significant alcohol consumption. DNA was extracted from swabs and the V1 region of the 16S rRNA gene was PCR amplified and sequenced using the lon Torrent PGM platform, generating 3.7 million high quality reads. DNA sequences were clustered and OTUs were assigned using the ARB SILVA database and Qiime.
\end{abstract}

Results: We found no differences in species diversity and evenness among the groups. However, we found a significant decrease in species richness in only smokers and in smokers/drinkers when compared to controls. We found that Neisseria abundance was significantly decreased in both groups when compared to controls. Smokers had significant increases in Prevotella and Capnocytophaga and reductions in Granulicatella, Staphylococcus, Peptostreptococcus and Gemella when compared to the two other groups. Controls showed higher abundance of Aggregibacter, whilst smokers/ drinkers had lower abundances of Fusobacteria. Samples from only smokers clustered closer together than to controls and smokers/drinkers, and also had a significant reduction in inter-group dissimilarity distances, indicating a more homogenous group than controls.

Conclusions: Our results indicate that the continued use of tobacco or alcohol plus tobacco significantly reduces bacterial richness, which apparently leads to a reduction in inter-group variability, turning the respective biofilms into a more homogenous microenvironment in terms of bacterial community composition, with possible consequences for human oral diseases.

Keywords: Alcohol, Tobacco, Microbiome, Oral cavity, 16S rRNA

\section{Background}

Each day nearly 4,000 people younger than 18 years old smoke their first cigarette, a quarter of which will become addicted [1,2]. Cigarette smoking has been linked to acute respiratory tract infections $[3,4]$ and lung cancer [5], among other diseases [6] and on average, smokers die

\footnotetext{
* Correspondence: emmanuel@usp.br

'Laboratory of Medical Genomics, Centro Internacional de Pesquisa, AC Camargo Cancer Center, São Paulo, SP, Brazil

${ }^{8}$ Laboratory of Neurosciences (LIM27), Institute of Psychiatry, Faculdade de Medicina, Universidade de São Paulo, São Paulo, SP, Brazil

Full list of author information is available at the end of the article
}

10 years before non-smokers [7]. As a consequence, it is estimated that the annual number of tobacco-related deaths will grow from about 6 million today to about 8 million in 2030 [8]. The negative effects of alcohol ingestion are not far behind. Global prevalence rates of alcohol dependency or chronic alcohol use were estimated to range from $0-16 \%$ in 2002 [9]. A study estimated that the net effect of alcohol consumption on health was responsible for $3.8 \%$ of all global deaths and $4.6 \%$ of global disability-adjusted life-years [10]. Chronic alcohol use has been linked to liver cirrhosis, liver cancer and several other 
diseases [11]. In 2002, 1.3 billion people were estimated to have smoked tobacco, whilst 2 billion were estimated to have consumed alcohol [9]. Unfortunately, when combined, alcohol and tobacco have an even worse impact on health, and studies have shown that tobacco users are more prone to use alcohol and vice versa [12]. Together, these drugs account for $12 \%$ of worldwide mortality rates [9], creating a huge global economic burden [13-15], as they increase the risk of several diseases [9], including oral cancer [16].

The use of cigarettes by adults, as well as passive smoke exposure in children, has been associated with increased carriage of pathogenic organisms in the upper airways [17]. It has been reported that substances present in tobacco smoke alter the charge and other properties of oral epithelial cell surfaces, allowing the growth of certain pathogenic bacteria [18]. Cigarette smoke also affects the survival of specific bacterial species isolated from the oral cavity, inhibiting some species of Neisseria and gram-positive bacteria such as Staphylococcus aureus and Streptococcus pneumonia [19]. However, little is known about the effects of chronic use of alcohol and tobacco on the oral microbiome.

The human oral cavity harbors more than 700 different bacterial taxa, possessing relevant quantitative (microbial richness) and qualitative (microbial community composition) differences between individuals [20-22]. Also, oral microbial profiles tend to show patterns of relative intraindividual stability over time [23]. Bacterial communities differ between healthy and diseased oral cavities and, as they can cause or prevent infections, this may have a significant impact on general health $[22,24]$. As most of the microbiome cannot be grown in vitro $[25,26]$, community profiling approaches based on the amplification of marker genes of bacterial DNA derived from samples of interest have become a popular way of surveying microbial communities. With the recent development of highthroughput sequencing technologies, the investigation of microbial communities using the 16S rRNA gene as a marker has become the standard method for profiling microbial communities in various ecological niches.

In the present study we investigate the effects of the chronic use of alcohol and tobacco over the oral microbiome, in terms of diversity and composition, using the 16S rRNA gene as a target and the Ion Torrent Personal Genome Machine (PGM) platform for high throughput sequencing. To our knowledge, this is the first large-scale study focused on evaluating the effects that chronic use of alcohol and tobacco have over the microbiome of human oral biofilms.

\section{Methods}

\section{Subjects}

A total of twenty-two subjects were recruited and signed an informed consent form prior to sample collection. A questionnaire was given to all subjects to evaluate general characteristics including oral health, oral hygiene habits, alcohol and tobacco use and others (Table 1). Subjects were examined by stomatologists and/or dentists and allocated to one of the following groups:

Smokers/Drinkers (SD) $(\mathrm{n}=7)$ : subjects belonging to this group were recruited at the Alcohol and Tobacco Abuse Group at the Institute and Department of Psychiatry, part of the São Paulo Medical School (FMUSP), in São Paulo, Brazil. All individuals in this group reported the use of at least 20 cigarettes/day with a regular smoking history of at least 10 years. These individuals also reported daily drinking habits $(>1 \times /$ day, $>3$ drinks/occasion) and a regular drinking history of at least 10 years.

Controls $(C)(n=9)$ : All subjects belonging to this group were healthy individuals recruited at the cancer screening campaigns at the AC Camargo Cancer Center, also in São Paulo, Brazil. Individuals from this group never smoked cigarettes or any other tobacco-related derivatives, reported no or very low consumption of alcohol, defined as: participants reporting the consumption of only lowethanol beverages ( $<5 \%$ ethanol), in small doses (maximum of 1 drink/occasion), with a drinking history inferior to 3 years and absence of daily drinking during this period. None of these individuals used alcohol-containing mouthwash solutions.

Smokers $(S)(n=6)$ : All subjects belonging to this group were recruited at the cancer screening campaigns at the AC Camargo Cancer Center. These individuals reported a regular smoking history of at least 20 cigarettes/day for the last 10 years and maintained their smoking habits prior to sample collection. Regarding ethanol consumption as an excluding aspect, the same criteria as the control group was adopted, including mouthwash use.

\section{Additional exclusion criteria for all groups}

Additional exclusion criteria for all subjects were as follows: 1) subjects diagnosed with cancer; 2) subjects reporting the use of antibiotics within the last 3 months prior to sample collection; 3 ) subjects reporting residence outside of the city of São Paulo in the past 5 years; 4) presence of comorbidities such as HIV, diabetes and ischaemic heart disease; 5) individuals younger than forty years of age and 6) presence of malignant or potentially pre-malignant oral lesions, such as leukoplakia or erythroplakia.

This study was approved by the ethics in research committees of AC Camargo Cancer Center (protocol 1459/10) and University of São Paulo (protocol 0895/11).

\section{Sample collection and DNA extraction}

In order to sample bacteria from the oral biofilm, we swabbed the following oral mucosal surfaces of each individual: the dorsum and the laterals of the tongue, the floor of the mouth and the buccal mucosa. Bacterial samples were collected from these areas using Sterile Foam Tipped 
Table 1 Subject characteristics

\begin{tabular}{|c|c|c|c|c|}
\hline Demographics & $C(n=9)$ & SD $(n=7)$ & $S(n=6)$ & P-Value \\
\hline Average age (years) & $58.11 \pm 8.28$ & $59.86 \pm 3.39$ & $56.67 \pm 2.49$ & 0.8458 \\
\hline Gender & $\mathrm{F}: 1 / \mathrm{M}: 8$ & $\mathrm{~F}: 1 / \mathrm{M}: 6$ & F:1/M:5 & 0.9522 \\
\hline Average height (m) & $1.71 \pm 0.09$ & $1.72 \pm 0.07$ & $1.69 \pm 0.02$ & 0.6845 \\
\hline Average weight (kg) & $80 \pm 11.94$ & $72.29 \pm 4.99$ & $79.66 \pm 5.95$ & 0.467 \\
\hline Average BMI & $27.44 \pm 4.11$ & $24.26 \pm 1.13$ & $27.72 \pm 1.49$ & 0.2433 \\
\hline Average cigarettes/day & 0 & $18.43 \pm 4.07$ & $24.5 \pm 3.49$ & $0.00022^{*}$ \\
\hline Average alcohol intake/day $(\mathrm{mL})$ in the last 6 months & $0.9 \pm 0.7$ & $41.3 \pm 14.3$ & $0.01 \pm 0.01$ & $0.004^{*}$ \\
\hline Estimated total drinks $†$ & $6.2 \pm 2.7$ & $1668.9 \pm 598.8$ & $0.44 \pm 0.44$ & $0.004^{*}$ \\
\hline \multirow[t]{2}{*}{ Daily mouthwash use } & Yes (1) & Yes (3) & Yes (1) & \multirow[t]{2}{*}{0.3132} \\
\hline & No (8) & No (4) & No (5) & \\
\hline \multirow[t]{3}{*}{ Tooth cleaning frequency (per day) } & $1 \times(0)$ & $1 \times(1)$ & $1 \times(1)$ & \multirow[t]{3}{*}{0.7669} \\
\hline & $2 \times(4)$ & $2 \times(3)$ & $2 \times(2)$ & \\
\hline & $3 \times(5)$ & $3 \times(3)$ & $3 \times(3)$ & \\
\hline \multirow[t]{2}{*}{ Self reported gingival bleeding } & Yes (4) & Yes (1) & Yes (3) & \multirow[t]{2}{*}{0.9676} \\
\hline & No (5) & No (6) & No (3) & \\
\hline \multirow[t]{2}{*}{ Use of dentures } & Yes (4) & Yes (4) & Yes (3) & \multirow[t]{2}{*}{0.8858} \\
\hline & No (5) & No (3) & No (3) & \\
\hline \multirow[t]{4}{*}{ Frequency of dentist appointments } & 1×/year (5) & $1 \times /$ year $(2)$ & $1 \times /$ year $(1)$ & \multirow[t]{4}{*}{0.5425} \\
\hline & $1 \times / 2-5$ years $(1)$ & $1 \times / 2-5$ years $(2)$ & $1 \times / 2-5$ years $(1)$ & \\
\hline & $1 \times / 5$ years $(2)$ & $1 \times / 5$ years $(1)$ & $1 \times / 5$ years $(2)$ & \\
\hline & Never (1) & Never (2) & Never (2) & \\
\hline
\end{tabular}

Values are given as average \pm standard error. Height is given in meters $(\mathrm{m})$, weight in kilograms $(\mathrm{kg})$ and average daily alcohol intake is shown in milliliters ( $\mathrm{mL}$ ) per day. † Estimated total drinks = (average alcohol intake/day $(\mathrm{mL})) \times($ years drinking). C: controls; SD: smokers/drinkers; S: only smokers; F: females; M: males; BMI: body mass index. Statistically significant differences $(p<0.05)$ are indicated $(*)$ by the Kruskal-Wallis Test.

Applicators and transferred to FTA elute cards, both from Whatman (Maidstone, UK), where DNA was preserved after chemical lysis of the cells. In order to obtain a better representation of the whole oral mucosa in each sample, we collected four perforations $\left(7 \mathrm{~mm}^{2}\right.$ each) from different areas of each card and then eluted each one in $30 \mu$ of sterile water by incubating for $25 \mathrm{mins}$ at $95^{\circ} \mathrm{C}$, according to the manufacturer's recommendations. These four elutions from a single subject were pooled together and used as template DNA for subsequent procedures.

PCR amplification and sequencing of the V1-region of the 16S rRNA gene

PCR amplification

We amplified the eluted bacterial DNA using a previously described set of degenerated primers [27] which cover the V1-region of the 16S rRNA gene (F: 5 '-AGAGTTTGATC MTGGCTCAG-3' and R: 5' -TTACTCACCCGTICGCCR CT-3'), generating $\sim 113$ bp amplicons, compatible with the sequencing platform. For a better representation of the bacterial DNA, we performed a total of six simultaneous PCR amplifications for each subject. PCR mixes consisted of $2 \mu \mathrm{M}$ of each primer, $5 \mu \mathrm{l}$ of the $2 \mathrm{X}$ high-fidelity Taq DNA polymerase master-mix (Roche, Mannheim, Germany) and
$2 \mu \mathrm{l}$ of eluted DNA in a final volume of $10 \mu \mathrm{l}$. The cycling conditions included an initial denaturation step $\left(94^{\circ} \mathrm{C}\right.$ for 2 mins) followed by 35 cycles at $94^{\circ} \mathrm{C}$ for 15 secs and $60^{\circ} \mathrm{C}$ for 30 secs and a final extension step at $72^{\circ} \mathrm{C}$ for 5 mins.

\section{Sequencing}

We evaluated $2 \mu \mathrm{l}$ of the amplification products in $8 \%$ silver-stained polyacrilamyde gels [28] and quantified the amplicons of each individual using a Qubit high-sensitivity double stranded DNA kit (Qiagen, Crawley, UK). The amplicons were purified using AMPure XP beads (Beckman Coulter, Indianapolis, USA), end-repaired and Ion Torrent adaptors with $10 \mathrm{bp}$ barcodes were ligated to their ends using proprietary kits (Life Technologies, Carlsbad, USA). For emulsion PCR we pooled together equimolar amounts of amplicons from each sample, as indicated by a Bioanalyzer High Sensitivity chip (Agilent Technologies, Santa Clara, USA). All samples were sequenced on the Ion Torrent PGM platform (Life Technologies).

\section{Sequence analysis}

\section{Sequence filtering}

Sequences processed by the latest version of the Ion Torrent server (version 3.6.2) were used as input into the 
Qiime (Quantitative insights into microbial ecology) software package (Version 1.6.0) [29]. We first removed sequences that had an average quality score $<20$, then identified barcodes used for subject-assignment accepting no more than 2 mismatches and discarded sequences smaller than $30 \mathrm{nt}$ or greater than $200 \mathrm{nt}$. PCR primers identified at the start or at the end of the reads, allowing a maximum of 1 mismatch, were trimmed. Sequences with no identifiable primers were discarded. After primer trimming we again removed all sequences under $30 \mathrm{nt}$. We used the remaining sequences for downstream analysis.

\section{Sequence clustering and OTU filtering}

After primer- and quality-filtering, we clustered the remaining sequences with $97 \%$ identity using UPARSE (implemented in USEARCH v7) [30], and picked the less dissimilar sequence of each cluster as its' representative. Chimeric sequences (and clusters) were identified using UCHIME [31] and the Broad Institute's chimera slayer database (version microbiomeutil-r20110519) and excluded. We then used the Qiime interface to perform BLAST [32] analysis (using default parameters, with a maximum e-value of 0.001) against the formatted SILVA 111 SSU database [33] for taxonomic rank assignment of each sequence and, subsequently, to each operational taxonomic unit (OTU). In order to decrease PCR- and sequencingderived errors, OTUs that were not present in at least $25 \%$ of all samples, had less than three reads, or had no taxonomic classification, were discarded from further analysis.

\section{Data normalization}

To investigate differences in phyla and genera abundance between the three groups, raw counts were normalized using the following normalization method [34]:

Normalized count $=\log _{10}[($ raw count $/$ number of sequences in that sample $)$ $x$ average number of sequences per sample +1$]$

\section{Alpha diversity}

To investigate species diversity, richness and evenness, we used the same sequence sampling depth for all samples by rarefying the OTU table. Rarefaction curves, the ShannonWeaver diversity index [35] and equitability for species evenness were calculated using Qiime. Observed species richness and richness estimators Chao1 [36] and ACE [37] were calculated using the $\mathrm{R}$ (Open sourced statistical software, Vienna, Austria - Version 3.0.1) Phyloseq package (Version 1.4.5) [38].

\section{Beta diversity}

The Euclidean distance matrix, the coordinates for the Principal Coordinate Analysis (PCoA), and the intra- and inter-dissimilarity indexes were calculated using Qiime.

\section{Statistical analysis}

Kruskal-Wallis tests were performed to compare mean similarities between controls, smokers/drinkers and only smokers for phyla and genera log abundances. P-values were corrected for multiple testing [34] using: ( $t * \mathrm{p}) / \mathrm{R}$; where $\mathrm{t}=$ total number of taxa tested; $\mathrm{p}=$ raw $\mathrm{p}$-value and $\mathrm{R}=$ sorted rank of the taxon. Kruskal-Wallis tests were also performed on categorical data of the subject's characteristics using R. Independent two tailed T tests were performed on different alpha diversity metrics between pairs of the three groups.

\section{Results and discussion Subjects}

Among the 22 subjects studied here, 19 were males (8 controls, 6 smokers/drinkers and 5 only smokers) and 3 females (one in each group). The composition of the groups showed no statistical differences in terms of age, gender, weight and height. Whereas the self-reports of general oral health, oral hygiene habits, gingival bleeding, annual visits to the dentist and others were also similar among the groups, as expected, differences in alcohol and tobacco consumption showed to be statistically significant. Table 1 shows the general sample characteristics, including pvalues to demonstrate, if present, statistical differences between the three groups. Two subjects, one belonging to the only smokers group and one to the smokers/drinkers group were positive for hepatitis $\mathrm{C}$, whilst another subject from the smokers/drinkers group was hypertensive. As alpha and beta diversity analysis indicated that these samples were not significantly different from others in their respective group (data not shown), they were included in downstream analysis. With regards to the use of other drugs, only one subject that belonged to the only smokers group admitted to the use of cocaine but had not used the drug for over a year, and was therefore also included in the study.

\section{Sequence analysis \\ Sequence filtering}

After size, quality and primer trimming, a total of 3,843,174 sequences remained, with an average of 174,689 sequences/ sample and a mean sequence length of $72 \pm 4.9 \mathrm{nt}$ (standard deviation) after primer trimming and barcode removal.

\section{Sequence clustering and OTU filtering}

When all individuals were considered, a total of 2,931 clusters were obtained. Twenty-one $(0.7 \%)$ of these were removed after being identified as chimeras by UCHIME, and $112(3.8 \%)$ had no assigned taxonomy. After filtering to remove OTU clusters with less than 3 sequences and not present in at least $25 \%$ of all samples, 1,849 OTUs remained. 


\section{Beta diversity between controls, only smokers and smokers/drinkers \\ Beta diversity}

Using oral wash and broncheoalveolar lavage samples, a previous study found significant differences in bacterial communities when comparing smokers and non-smokers, suggesting that tobacco may significantly affect the oral microbiome [39]. In our study we first sought to evaluate if the 16S rRNA data generated would be capable of clustering individuals according to their use (or not) of alcohol/tobacco. We opted for the binary Euclidean distance metric due to the size of the $16 \mathrm{~S}$ rRNA hypervariable region. Figure 1A shows the results of the PCoA plot using the distance matrix obtained by the binary Euclidean metric, demonstrating that 16S rRNA profiles clustered all individuals from the SD group together, as well as all samples from the $\mathrm{S}$ group. One of the control samples clustered closer to smokers/drinkers, whereas the other eight remaining control samples formed an independent group. Also, one of the smokers/drinkers samples clustered closer to only smokers, whilst the other six formed an independent group. The cladogram in Figure 1B indicates that all samples of the control group (except sample C01 which clustered closer to smokers/drinkers in the PCoA plot) belonged to the same main branch and that samples from smokers/drinkers were closer to only smokers than to controls. Also, all members of the only smokersgroup clustered in the same tree branch. This consistent clustering suggests that alcohol and principally tobacco might influence the growth of certain bacterial species and may impact the normal abundance of bacteria commonly found in the oral cavity's mucosa. It also suggests that smokers/drinkers have bacterial communities more similar to that of only smokers, rather than to controls.

\section{Intra- and inter-group similarity analyses}

We then used the binary Euclidean metric to investigate if bacterial communities were more similar within each sample group or between groups, i.e. an intra-group $v s$ inter-group dissimilarity analysis. Figure 2 illustrates our findings, demonstrating that samples from the only smokers group were statistically more similar, in terms of bacterial communities, between themselves than between samples from the two other groups. This suggests that tobacco may play a key role on quantitative/qualitative bacterial alterations, turning the only smokers sample group into the least dissimilar. Unfortunately we were not able to recruit a reliable group of alcohol-drinkers that do not use tobacco in order to properly address the role that ethanol alone would have over the oral mucosa's microbiome. However, our analyses also demonstrate that the control group was the most dissimilar; evidencing how variable and diverse the healthy oral microbiome is between samples, especially in the absence of substances that appear to reduce the abundance of certain bacteria.

A recent study showed that even with the considerable intra- and interpersonal variation in the human microbiome, this variation could not be partitioned into community types that are predictive of each other, and were probably the result of life-history characteristics [40], which may include tobacco and/or alcohol consumption. Our results suggest that the chronic use of alcohol and principally tobacco imposes a constraint on the abundance of certain bacteria found in the oral cavity of subjects that do not ingest these substances on a regular and heavy basis, turning the otherwise healthy and diverse bacterial collection into a more homogeneous and restrict environment. Whereas it has been suggested that environment and diet do not significantly influence the composition of the

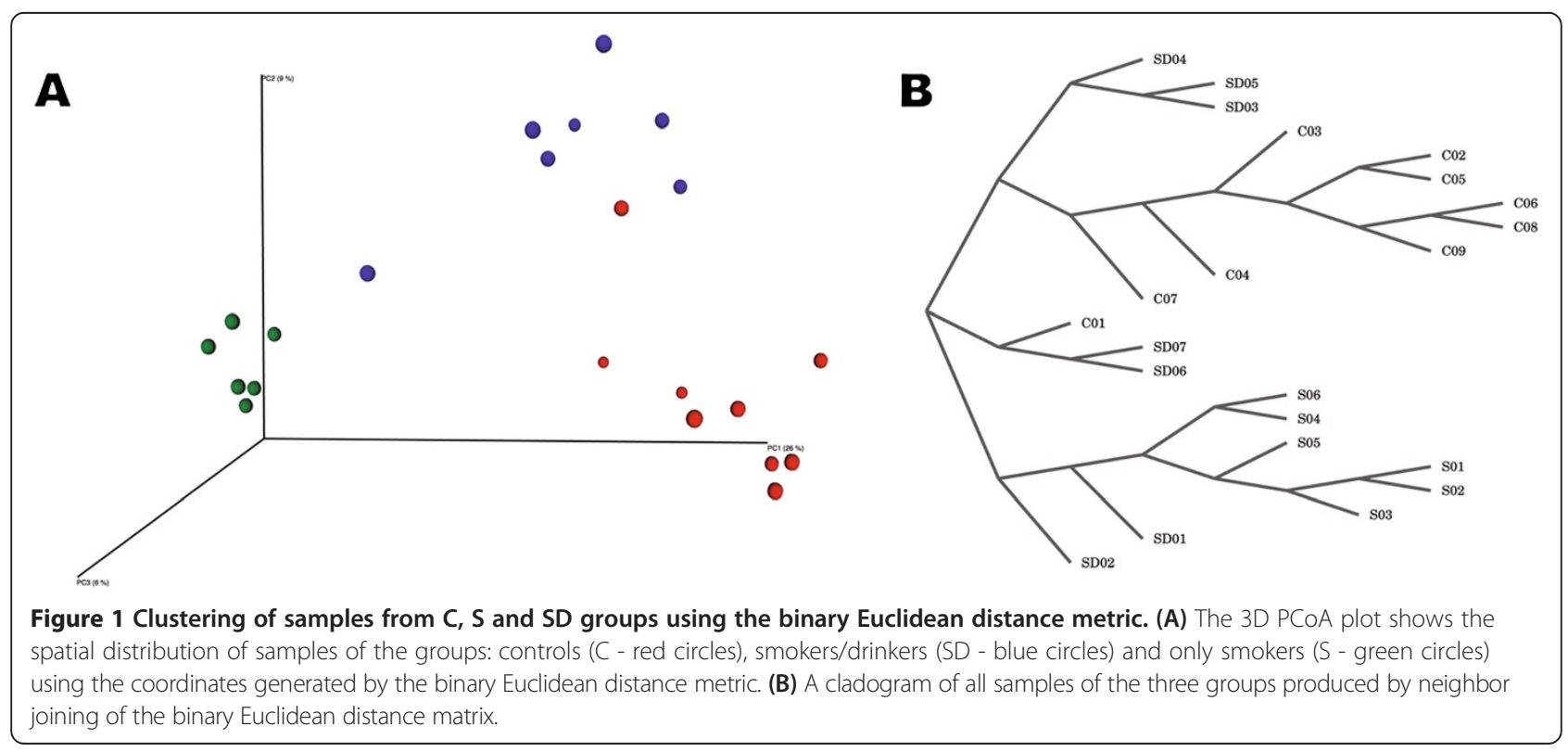




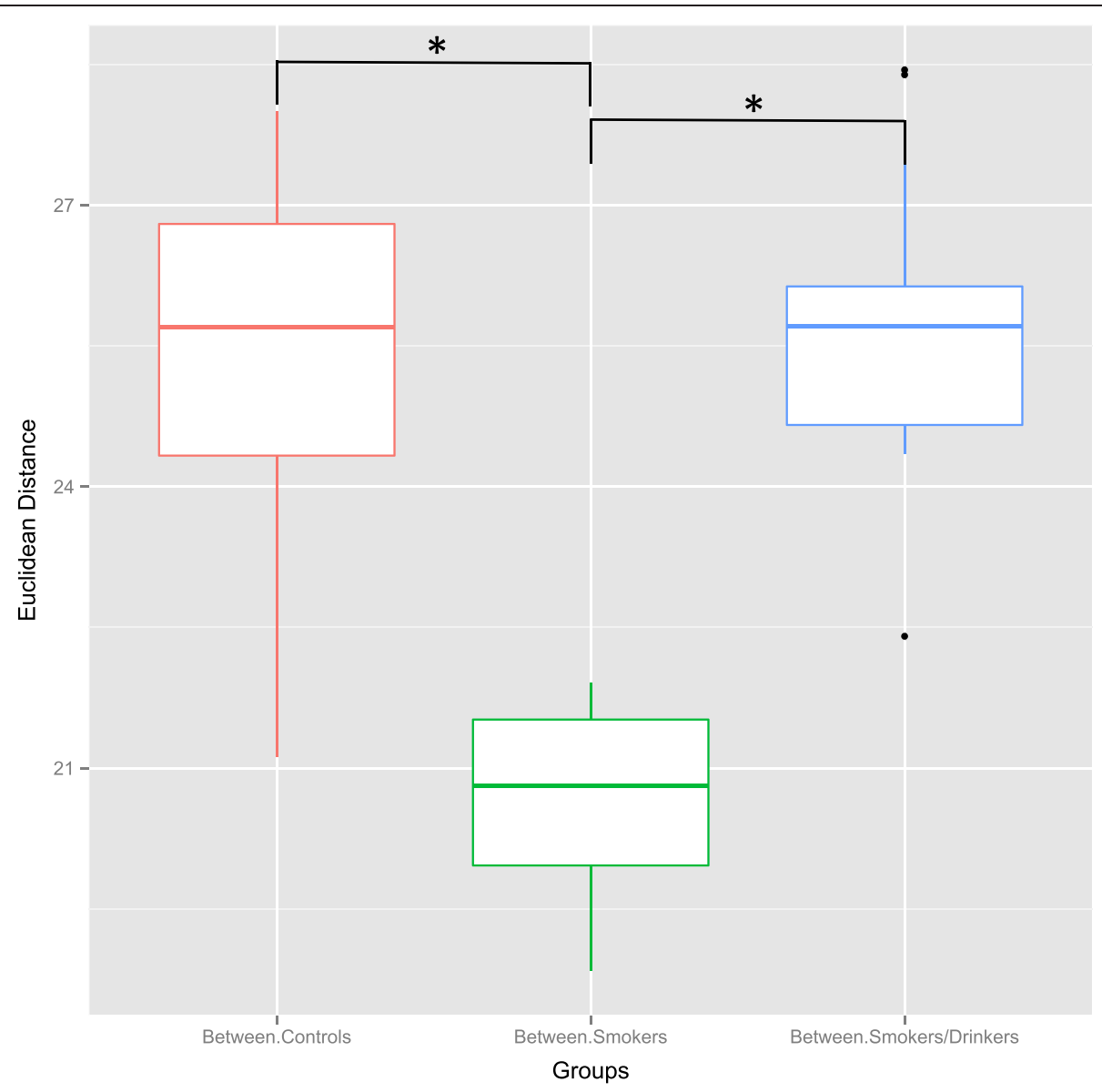

Figure 2 Inter- and intra-group distances between C, S and SD groups. The boxplot shows the average binary Euclidean distance when comparing intra and inter group dissimilarity. Significant comparisons between mean distances, as evidenced by a parametric T-test, are indicated $\left(^{*}=p<0.05\right)$.

oral microbiome [21], our data suggests that the consumption of alcohol and tobacco may be one of the primary determinants of microbial composition.

\section{Changes in alpha diversity caused by alcohol and tobacco Species richness}

In order to measure species richness in the three groups we used the number of distinct OTUs observed in each sample when the same sequence sampling depth was applied at two different OTU-abundance thresholds (Figure 3). Saturation was not achieved when all OTUs represented by at least three reads were considered. This is likely to be derived from the combined effect of a still low coverage of very rare OTUs (those with frequencies below $0.03 \%$, i.e. less than three reads $/ 10,040$ reads), together with PCR-amplification and/or sequencing artifacts. On the other hand, using this cutoff of at least three reads, which might underestimate very rare bacteria and precludes the representation of microorganisms nonamplifiable by this set of "universal primers", as well as bacteria that cannot be discriminated on the basis of sequences derived from the variable region 1 (V1) of the
$16 \mathrm{~S}$ rRNA gene, we clearly see that the microbiome of all groups exceeds 300 distinct OTUs (Figure 3A), indicating a rich community. Indeed, bacterial communities of the oral cavity are one of the most complex across all body sites, second only to the colon [41]. When we considered a more stringent OTU abundance threshold (Figure 3B), a saturation trend for OTUs with at least 100 reads ( $1 \%$ of the sequences used for each subject) could be seen and indicates the presence of at least 200 OTUs with frequencies above this threshold (Figure 3B). When considering OTUs with at least three reads, we observed that the average species richness of controls was about $35 \%$ and $17 \%$ larger than that of only smokers and smokers/drinkers, respectively (Figure 3A-B). When the same sequencing depth was used to evaluate species richness between pairs of the three groups, using three distinct metrics, we observed a significant decrease in species richness for only smokers when compared to controls. A significant reduction in OTU richness between controls and smokers/drinkers was also found at the sampling depth of 142,161 reads. The reduction in richness (in both SD and $\mathrm{S}$ when compared to $\mathrm{C}$ ) was observed when using the 

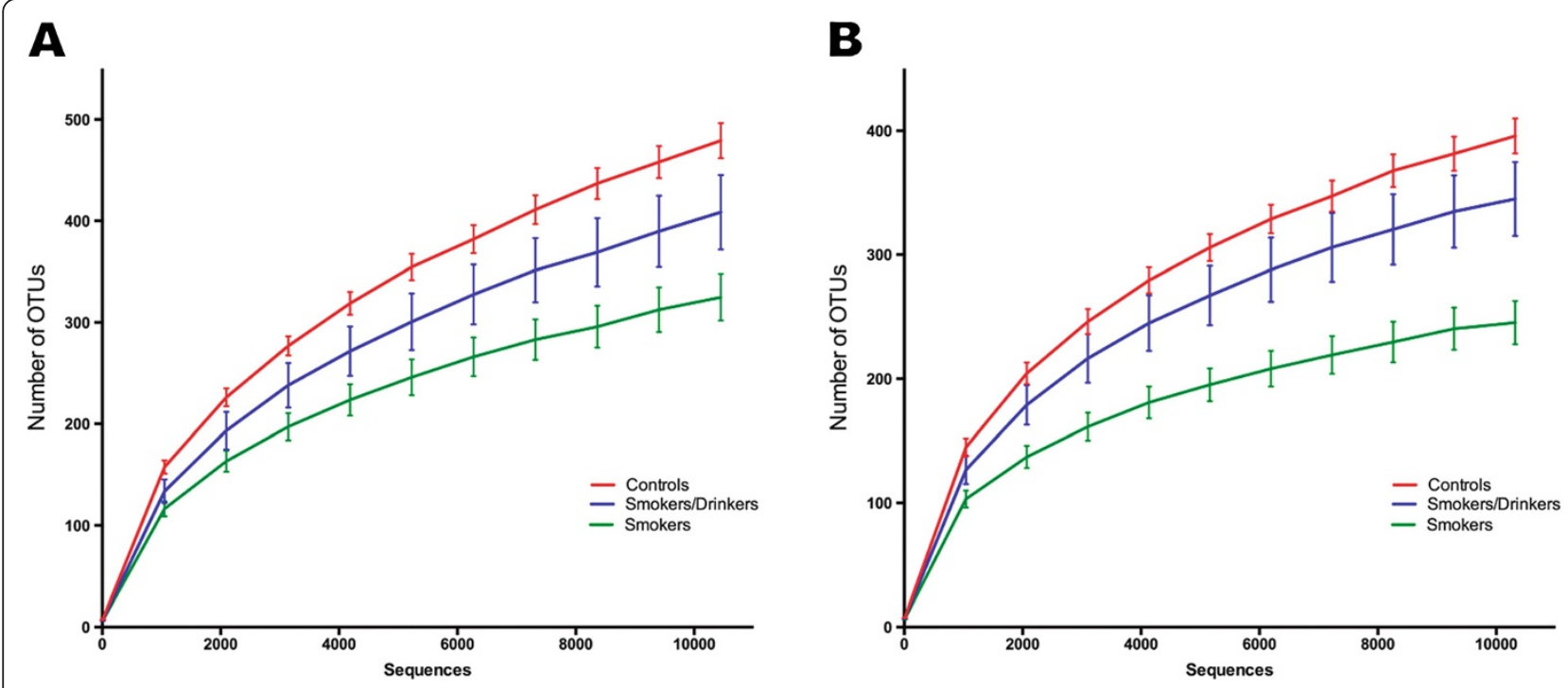

Figure 3 Rarefaction curves at two different OTU abundance thresholds. For each number of sequences the number of distinct OTUs was calculated; OTUs represented by one or two sequence reads were not considered. The distinct charts represent the rarefaction curves produced when we considered: (A) OTUs represented by at least 3 reads (or 0.03\% of samples); (B) OTUs with a minimum of 100 reads (1\%). Lines represent the average number of distinct OTUs \pm standard error (S.E).

Observed Richness (only smokers p-value $=0.0005$; smokers/ drinkers $\mathrm{p}$-value $=0.0446)$ and also for richness estimators Chao1 (only smokers $\mathrm{p}$-value $=0.0013$; smokers $/$ drinkers $\mathrm{p}$-value $=0.0465)$ and ACE (only smokers p-value $=0.0009$; smokers/drinkers p-value $=0.0474)$ (Figure 4). Surprisingly, we found no statistical difference in species richness between only smokers and smokers/drinkers using any of these metrics $(\mathrm{p}>0.05)$.

\section{Species evenness}

We evaluated species evenness through the use of equitability (mean C: 0.58; mean SD: 0.55; mean S: 0.55, where

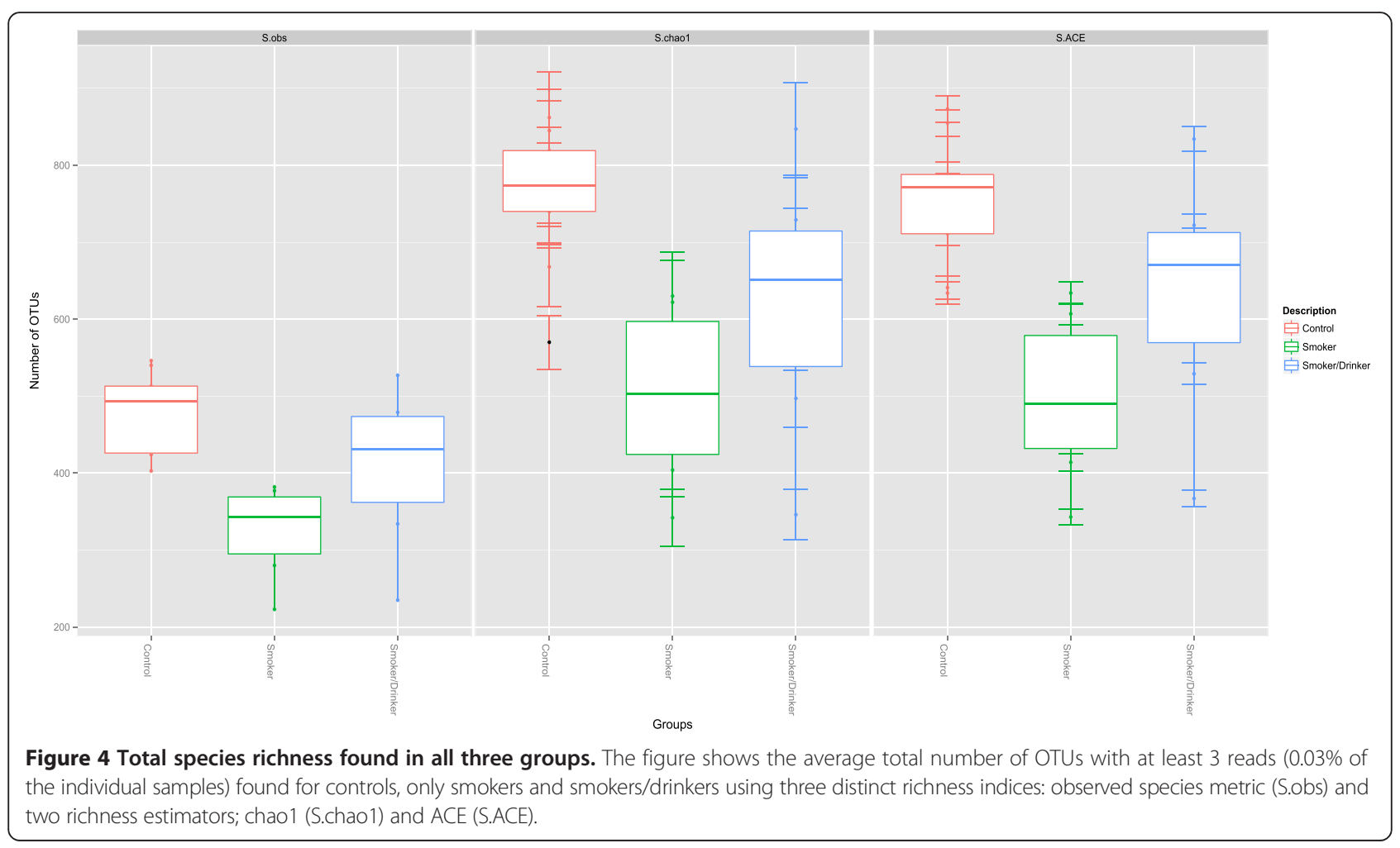


1 indicates complete equitability) and found no statistical difference in species evenness between these three groups ( $p>0.05$ ) (Table 2). This suggests, for all groups, the presence of numerous species with low overall abundance and few highly abundant species. Data from the Human Microbiome Project showed that, within a cohort, bacterial members present in low abundance, low ubiquity or both, may contribute to high interpersonal variations of the microbiome [42], and this may apply here, by reducing microbial richness in only smokers and therefore reducing the dissimilarity between subjects of this group.

\section{Species diversity}

Also, we evaluated species diversity using the ShannonWeaver index and found no significant differences in diversity between each of the possible group pairs, but did see an increase in controls (mean C: 5.19, SD: 4.76 and S: 4.59). This suggests that the key difference in alpha diversity between controls, smokers/drinkers and only

\begin{tabular}{|c|c|c|c|c|c|}
\hline Samples & S.obs & S.chao1 & S.ACE & $\begin{array}{l}\text { Shannon- } \\
\text { Weaver Index }\end{array}$ & Equitability \\
\hline$\overline{\mathrm{C} 01}$ & 426 & 774 & 711 & 5.13 & 0.59 \\
\hline C02 & 546 & 845 & 873 & 5.49 & 0.6 \\
\hline $\mathrm{CO3}$ & 493 & 819 & 773 & 5.65 & 0.62 \\
\hline $\mathrm{CO} 4$ & 403 & 570 & 641 & 4.71 & 0.54 \\
\hline C05 & 483 & 749 & 765 & 4.76 & 0.54 \\
\hline C06 & 494 & 740 & 771 & 5.33 & 0.6 \\
\hline $\mathrm{CO}$ & 424 & 668 & 634 & 4.47 & 0.52 \\
\hline C08 & 513 & 777 & 788 & 6.01 & 0.66 \\
\hline CO9 & 540 & 862 & 855 & 5.17 & 0.57 \\
\hline S01 & 340 & 522 & 494 & 4.64 & 0.56 \\
\hline SO2 & 280 & 404 & 414 & 4.45 & 0.55 \\
\hline S03 & 223 & 342 & 343 & 3.63 & 0.47 \\
\hline SO4 & 345 & 485 & 486 & 5.16 & 0.61 \\
\hline S05 & 377 & 622 & 634 & 4.8 & 0.56 \\
\hline S06 & 382 & 630 & 607 & 4.88 & 0.56 \\
\hline SD01 & 235 & 346 & 367 & 3.62 & 0.47 \\
\hline SD02 & 468 & 729 & 703 & 5.34 & 0.6 \\
\hline SD03 & 431 & 651 & 670 & 5.29 & 0.6 \\
\hline SD04 & 479 & 700 & 722 & 4.87 & 0.56 \\
\hline SD05 & 527 & 847 & 834 & 5.26 & 0.59 \\
\hline SD06 & 334 & 497 & 529 & 3.94 & 0.47 \\
\hline SD07 & 390 & 580 & 610 & 5.02 & 0.59 \\
\hline
\end{tabular}

The table shows the number of OTUs with regards to species richness (observed, Chao1, ACE), species evenness (equitability) and diversity (Shannon-Weaver Index) for each sample using the sample sequence sampling depth $(10,040$ sequences). smokers lies solely in species richness. A previous study found a more diverse microbiome in the oropharynx of smokers than non-smokers [43], however it was restricted to tobacco-induced effects and no alcohol-drinking habits were reported. Another study evaluated the effects of chronic alcohol feeding on the intestinal microbiome of mice [44]. In this case, a reduction in species richness was observed along three time-points in alcohol-fed versus pellet-fed animals, and this decrease showed a negative correlation between species richness and the cumulative time mice were alcohol-fed. Another recent work [45] also found a reduction in species richness, this time in the oral biofilms covering the teeth of rats that received 30-days of a $20 \%$ ethanol-containing diet. Besides technical limitations due to the approach used for bacteria detection and quantification in the biofilms of rat teeth (restricted to probes for only 33 bacteria of the human oral microbiome) these authors were able to demonstrate a significant association between alcohol consumption and lower levels of bacterial richness. A reduction in microbial diversity has been associated with other diseases such as irritable bowel syndrome [46], eczema [47], Crohn's disease [48], obesity [49] and recurrent Clostridium difficileassociated diarrhea [50], evidencing the crucial role that commensal bacteria play on keeping pathogenic microbes at bay and maintaining general homeostasis.

The chronic and simultaneous use of alcohol and tobacco appears to significantly decrease species richness in the human oral microbiome, which could lead to dysbiosis, the unbalancing of the relative abundance of individual components of the microbiota compared to their abundance in health [51]. It has been hypothesized that dysbiosis could lead to a variety of diseases [23]. Our data indicates that tobacco, together or not with alcohol, affects the microbiome leading to reduced species richness, through a mechanism that probably includes pro- and anti-inflammatory molecules [52]. To our knowledge, the work presented here is the first to evaluate microbial diversity and composition in biofilms of the oral mucosa in groups of human subjects with regards to the simultaneous and chronic use of alcohol and tobacco and the use of tobacco alone.

\section{Eubacterial phyla and genera most affected by alcohol and tobacco Phyla log abundances}

The most abundant phyla found in all groups were (in decreasing order of abundance): Firmicutes, Actinobacteria, Proteobacteria, Bacteroidetes, TM7, Fusobacteria and Tenericutes (Figure 5A). At the phylum level, six out of 16 phyla were found to have significant differential log abundances between the three groups (Additional file 1: Table S1). Only smokers had an increase of Actinobacteria and Fusobacteria and a decrease of Firmicutes, Cyanobacteria, 


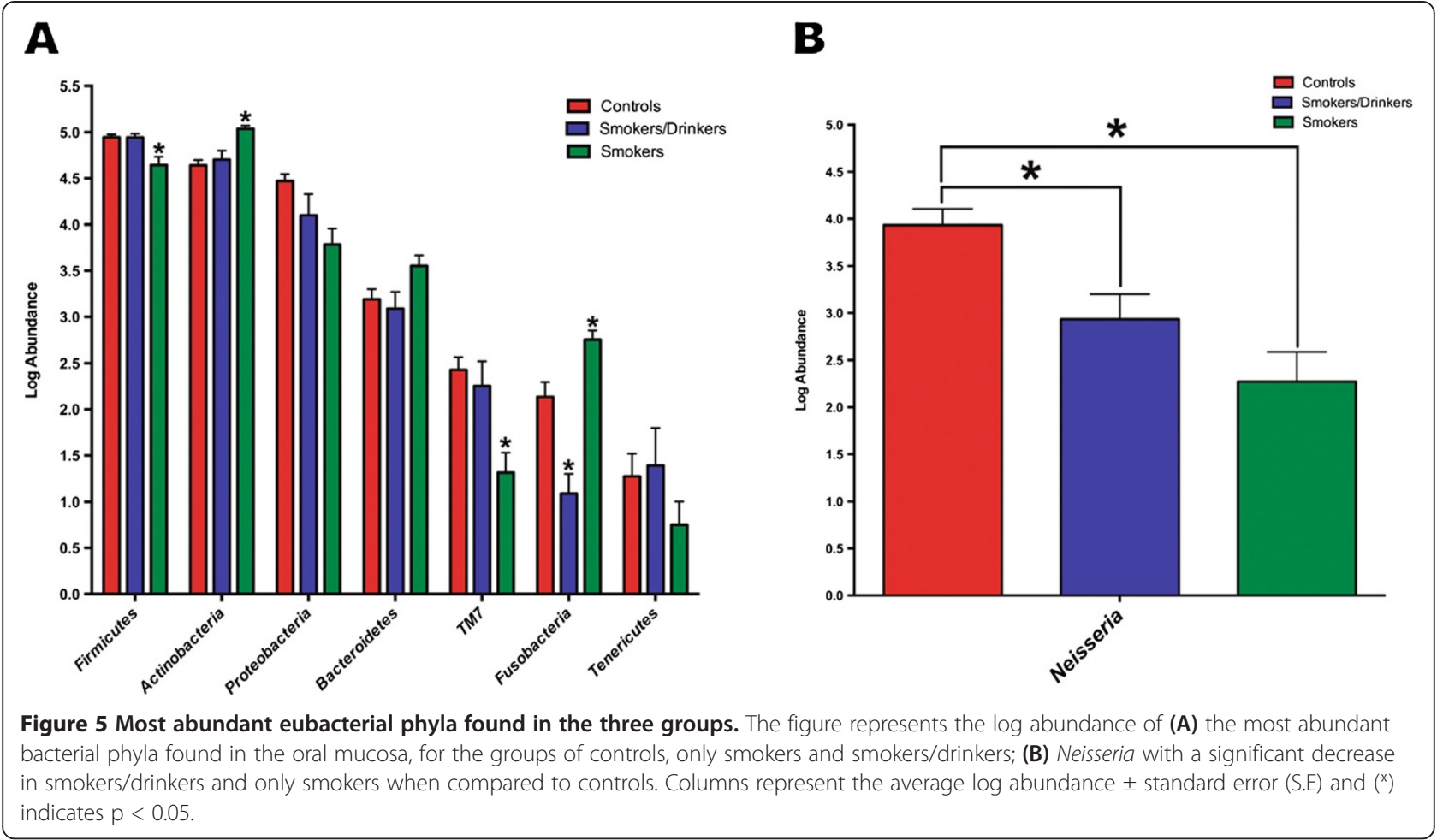

TM7 and BD1-5 when compared to the two other groups, whilst smokers/drinkers had a decrease of Fusobacteria. TM7 is a novel candidate bacterial division and previous studies have shown that microbes from this division are commonly found in the human oral flora at relatively low abundance, generally around 1\% $[53,54]$ and up to $8 \%$ [55] of the oral bacterial population, and a possible role of this phylum in periodontal disease has been previously suggested $[53,56]$. In only smokers, a significant reduction in log abundance of Proteobacteria could be observed (Kruskal-Wallis test, $\mathrm{p}=0.03$ ), but after correcting for multiple taxa testing, this decrease was no longer significant $(p=0.06)$. A reduction in the relative abundance of this phylum in broncheoalveolar-lavages of smokers when compared to non-smokers has been reported [39], pointing to a role that tobacco may play on reducing representatives of this phylum.

Genera log abundances. At the genus level, 47 out of the 279 (16.8\%) identified genera were found to have significant differential log abundances between the three groups (Additional file 2: Table S2). The most abundant genera found in controls were (in decreasing order of abundance): Veillonella, Actinomyces, Streptococcus, Haemophilus and Neisseria. In smokers/drinkers the most abundant genera were: Veillonella, Actinomyces, Rothia, Granulicatella and Streptococcus and in only smokers were: Actinomyces, Rothia, Veillonella, Streptococcus and Leucobacter. All these genera have been reported before as common residents of the oral cavity and of oral biofilms [20,24,57]. We found a significant decrease in log abundance of Neisseria in both only smokers and smokers/drinkers when compared to controls (Kruskal-Wallis test $\mathrm{p}=0.026$; Figure $5 \mathrm{~B}$ ), but found no difference in the log abundance of this genus between SD and S. Whereas Neisseria is one of the most dominant genera found in the larynx, including laryngeal tumors [58], other studies have reported that the abundance of this genus is modulated by tobacco as its levels are reduced in the oral wash, oro-/nasopharyngeal swabs and bronchoscopic alveolar lavages of cigarette smokers [39,43,59].

Only smokers had significant increases in Prevotella and Capnocytophaga and decreases in Granulicatella, Staphylococcus, Peptostreptococcus and Gemella when compared to the other two groups. A significant decrease in abundance of Peptostreptococcus in smokers has been evidenced before [43], suggesting the susceptibility of this genus to smoke exposure. It is of interest to note that this particular reduction may be significant as several species belonging to this genus have shown to interfere in the growth of pathogenic bacteria in the upper respiratory tract [60]. Another genus that also seems to be modulated by smoking is Gemella, with a previous study also finding a decrease in the abundance of this genus [39]. In our analysis, the genus Porphyromonas, which has been shown to be increased in smokers $[39,43]$ and to have a role in periodontitis $[61,62]$, was also found to have higher abundances in only smokers (mean C: 0.67, SD: 0.29 and $\mathbf{S}: 1.8)$. However, after multiple taxa correction, 
the $\mathrm{p}$-value was no longer significant (Kruskal-Wallis test $\mathrm{p}$-value $=0.08$ ). Subsequent reports using $16 \mathrm{~S}$ rRNA sequence profiling of subgingival plaque identified an increase in several disease-associated organisms in smokers, including Parvimonas, Fusobacterium, Campylobacter, Bacteroides, Dialister, and Treponema spp. and a decrease in potential health-promoting taxa from the Veillonella, Neisseria, Streptococcus, and Capnocytophaga genera [63]. Aggregibacter levels were higher in controls, whilst smokers/drinkers had lower log abundances of Fusobacteria compared to $\mathrm{C}$ and $\mathrm{S}$ (Kruskal-Wallis test; p-values= 0.04417 and 0.02541 , respectively).

\section{Conclusions}

The study presented here has certain limitations that need to be considered. First we would like to point out that the sample size was relatively small $(\mathrm{N}=22)$ and limited to a set of individuals living in the city of São Paulo, Brazil. The study of larger cohorts, and a cohort composed of individuals that consume alcohol but do not smoke would be important for a better understanding of the individual effects of these substances. The study of individuals who have quit using alcohol and tobacco, as well as recent users, would be important to reveal how long a 'normal' microbiome persists and how long it takes to recover after the use of alcohol/tobacco. Besides the overall evaluation of oral hygiene habits and oral health status (Table 1), we did not check for the presence of periodontal disease. Our findings are also limited by the choice of PCR primers targeting the V1 region of the $16 \mathrm{~S}$ rRNA gene. In silico analysis indicated that, whereas this primer pair covers about $54.5 \%$ of the eubacterial phyla present in the ARB SILVA database, certain phyla are clearly under-represented, including Fusobacteria and Bacteroidetes. Also, whereas the DNA extraction protocol used here does not include the use of physical lysis (such as a bead beating step), which may improve the representation of certain bacteria (especially Gram-negative), and whilst PCR/sequencing artifacts may still persist, all these effects should be homogeneous for all groups and should not greatly affect our conclusions. A previous study [64] reported that a correction for the number of rRNA operons influences the distribution of bacterial phyla found in oceans. Considering that a) this operon normalization correction was optimized for larger 16S rRNA amplicons and b) the authors suggest that this correction would not greatly affect phylum distributions in the human microbiome, this correction was not implemented here. A recently described tool, named CopyRighter [65] showed to be capable of improving estimates of relative OTU abundances in human microbiome profiles after rRNA copynumber correction, albeit we have not investigated whether the use of this tool would affect our main conclusions.
Taking these limitations into consideration, we found a reduced bacterial richness in both only smokers and smokers/drinkers when compared to controls. To our knowledge, no studies have evaluated, up to now, if alcohol and tobacco affect the microbial composition of the human oral mucosa. In this study we found that bacterial communities from subjects who chronically use tobacco together or not with alcohol - cluster together and possess reduced species richness, suggesting that this substance may play a key role in reducing the oral cavity's bacterial richness. This reduced richness might have contributed to the significant decrease in dissimilarity observed between subjects of the only smokers group, showing to be the most homogenous in terms of bacterial communities. Species diversity and species evenness were not significantly different between any of the group pairs. Although, independent of the metrics used, all analyses indicated a higher species-richness in controls.

Our data indicates the presence of over 300 distinct bacterial OTUs in the human oral mucosa (Figure 3A). Our rarefaction curves show that, despite the limitations of the primer pair used and the size of the amplicons, for each individual that was sampled, we were able to represent OTUs that had a frequency of at least $1 \%$. At the phylum level, we found that 6 out of the 16 phyla were altered between individuals of the three groups, with only smokers possessing the greatest number of affected phyla. Only smokers had significant decreases in $\log$ abundance of Firmicutes and TM7 among others and significant increases in Fusobacteria and Actinobacteria.

We found a significant decrease in Neisseria abundance in both only smokers and smokers/drinkers, reinforcing evidence that points to a modulation of this genus's abundance by tobacco smoke. We found that two other genera, Gemella and Peptostreptococcus, were exclusively decreased in only smokers.

A recent study showed that modern dietary habits introduced in the early Neolithic period, including carbohydrate ingestion, affected the oral microbiome composition and diversity [66]. The authors conclude that the modern oral environment is likely to be less resilient to perturbations due to infection by pathogenic bacterial species or to dietary imbalances. Whereas the modern carbohydrate-rich diet appears to lead to a less diverse oral microbiome when compared to the hunter-gatherer diets, our results indicate further microbiome perturbations led by alcohol and tobacco consumption. The evidences presented here suggest that microbial richness preservation- and restoration-strategies may represent effective novel approaches for the prevention and treatment of some aspects of alcohol and tobacco related diseases.

\section{Availability of supporting data}

Nucleic acid sequences are available at NCBI/GenBank/ SRA under accession number "SRP044345". 


\section{Additional files}

Additional file 1: Table S1. Phyla log abundance.

Additional file 2: Table S2. Differential genera log abundance.

\section{Abbreviations}

C: Controls; SD: Smokers/drinkers; S: Only smokers; PCoA: Principal coordinate analysis; OTU: Operational taxonomic unit; nt: Nucleotide; bp: Base pair; PCR: Polymerase chain reaction.

\section{Competing interests}

The authors declare no competing interests.

\section{Authors' contributions}

Conceived and designed the experiments: AMT, LPK, DNN, EDN; Performed the experiments: AMT, LBG, MA; Analyzed the data: AMT, GFR, JCS, DNN, EDN; Contributed reagents/samples/analysis tools: ALNF, FOGN, GFR, AGA JCS, LPK; Wrote or edited the manuscript: AMT, JCS, DNN, EDN. All authors read and approved the final manuscript.

\section{Acknowledgements}

We are grateful to the anonymous individuals who agreed to voluntarily participate in this study. The authors acknowledge the financial support received from Fundação Beneficente Alzira Denise Hertzog Silva (ABADHS), Fundação de Amparo à Pesquisa do Estado de São Paulo (FAPESP), The National Institute of Science and Technology in Oncogenomics (INCITO) and Coordenação de Aperfeiçoamento de Pessoal de Ensino Superior (CAPES). EDN and JCS are fellows of Conselho Nacional de Desenvolvimento Científico e Tecnológico (CNPq, Brazil).

\section{Author details}

${ }^{1}$ Laboratory of Medical Genomics, Centro Internacional de Pesquisa, AC Camargo Cancer Center, São Paulo, SP, Brazil. ${ }^{2}$ Curso de Pós-graduação em Oncologia, Fundação Antônio Prudente/AC Camargo Cancer Center, São Paulo, SP, Brazil. ${ }^{3}$ Universidad de Los Andes, Bogota, Colombia. ${ }^{4}$ Department of Head and Neck Surgery and Otorhinolaryngology, AC Camargo Cancer Center and National Institute of Science and Technology in Oncogenomics (INCITO), São Paulo, SP, Brazil. ${ }^{5}$ Department of Psychiatry, Universidade de São Paulo and of Faculdade de Medicina do ABC, São Paulo, SP, Brazil. ${ }^{6}$ Departmento de Bioquímica, Instituto de Química, Universidade de São Paulo, São Paulo, SP, Brazil. Virginia Bioinformatics Institute, Virginia Tech, Blacksburg, VA, USA. ${ }^{8}$ Laboratory of Neurosciences (LIM27), Institute of Psychiatry, Faculdade de Medicina, Universidade de São Paulo, São Paulo, SP, Brazil.

Received: 18 July 2014 Accepted: 23 September 2014

Published online: 03 October 2014

\section{References}

1. Substance Abuse and Mental Health Administration: Results from the 2010 National Survey on Drug Use and Health: National Findings. Rockville (MD): Office of Applied Studies; 2013. Accessed June $5^{\text {th }}$.

2. U.S. Department of Health and Human Services: Preventing Tobacco Use Among Youth and Young Adults: A Report of the Surgeon General. Atlanta: U.S. Department of Health and Human Services, Centers for Disease Control and Prevention, Office on Smoking and Health; 2012. Available at http:// www.cdc.gov/tobacco/data_statistics/sgr/2012/.

3. Aronson MD, Weiss ST, Ben RL, Komaroff AL: Association between cigarette smoking and acute respiratory tract illness in young adults. JAMA 1982, 248:181-183.

4. Turkeltaub PC, Gergen PJ: Prevalence of upper and lower respiratory conditions in the US population by social and environmental factors: data from the second National Health and Nutrition Examination Survey, 1976 to 1980 (NHANES II). Ann Allergy 1991, 67:147-154.

5. Boyle $P$, Maisonneuve P: Lung cancer and tobacco smoking. Lung Cancer 1995, 12:167-181.

6. Fagerström K: The epidemiology of smoking. Drugs 2002, 62(2):1-9.

7. Jha P, Ramasundarahettige C, Landsman V, Rostron B, Thun M, Anderson RN, McAfee T, Peto R: 21 st century hazards of smoking and benefits of cessation in the united states. N Engl J Med 2013, 368:341-350.
8. The Lancet: Tobacco control: when economics trumps health. Lancet 2013, 382:182.

9. World Health Organization, Substance Abuse, Global Burden of Disease. 2002. Available: http://www.who.int/substance_abuse/facts/global_burden. Accessed 30 July 2013

10. Rehm J, Mathers C, Popova S, Thavorncharoensap M, Teerawattananon Y, Patra J: Global burden of disease and injury and economic cost attributable to alcohol use and alcohol-use disorders. Lancet 2009, 373:2223-2233.

11. Rhem J, Shield KD: Global alcohol-attributable deaths from cancer, liver cirrhosis, and injury in 2010. Alcohol Res 2013, 35(2):174-183.

12. Bobo JK, Husten C: Sociocultural influences on smoking and drinking. Alcohol Res Health 2000, 24:225-232.

13. Cummings KM, Stiles J, Mahoney MC, Sciandra R: Health and economic impact of cigarette smoking in New York State, 1987-1989. N Y State J Med 1992, 92(11):469-473.

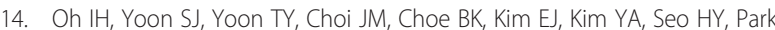
$\mathrm{YH}$ : Health and economic burden of major cancers due to smoking in Korea. Asian Pac J Cancer Prev 2012, 13(4):1525-1531.

15. Cai L, Cu W, He J, Wu X: The economic burden of smoking and secondhand smoke exposure in rural South-West China. J Asthma 2014, 51(5):515-521.

16. Soerjomataram I, deVries E, Pukkala E, Coebergh JW: Excess of cancers in Europe: a study of eleven major cancers amenable to lifestyle change. Int J Cancer 2007, 120:1336-1343.

17. Brook I, Gober AE: Recovery of potential pathogens in the nasopharynx of healthy and otitis media-prone children and their smoking and nonsmoking parents. Ann Otol Rhinol Laryngol 2008, 117:727-730.

18. El Ahmer OR, Essery SD, Saadi AT, Raza MW, Ogilvie MM, Weir DM Blackwell CC: The effect of cigarette smoke on adherence of respiratory pathogens to buccal epithelial cells. FEMS Immunol Med Microbiol 1999, 23:27-36.

19. Ertel A, Eng R, Smith SM: The differential effect of cigarette smoke on the growth of bacteria found in humans. Chest 1991, 100:628-630.

20. Paster BJ, Olsen I, Aas JA, Dewhirst FE: The breadth of bacterial diversity in the human periodontal pocket and other oral sites. Periodontol 2006, 42:80-87.

21. Nasidze I, Li J, Quinque D, Tang K, Stoneking M: Global diversity in the human salivary microbiome. Genome Res 2009, 4:636-643.

22. Lazarevic V, Whiteson K, Hernandez D, Francois P, Schrenzel J: Study of inter- and intra-individual variations in the salivary microbiota. BMC Genomics 2010, 11:523-534.

23. Costello EK, Lauber CL, Hamady M, Fierer N, Gordon Jl, Knight R: Bacterial community variation in human body habitats across space and time. Science 2009, 326:1694-1697.

24. Aas JA, Paster BJ, Stokes LN, Olsen I, Dewhirst FE: Defining the normal bacterial flora of the oral cavity. J Clin Microbiol 2005, 43:5721-5732.

25. Pei Z, Bini EJ, Yang L, Zhou M, Francois F, Blaser MJ: Bacterial biota in the human distal esophagus. PNAS USA 2004, 101:4250-4255.

26. Zhou X, Bent SJ, Schneider MG, Davis CC, Islam MR, Forney LJ: Characterization of vaginal microbial communities in adult healthy women using cultivation-independent methods. Microbiology 2004, 150:2565-2573.

27. Sundquist A, Bigdeli S, Jalili R, Druzin ML, Waller S, Pullen KM, El-Sayed YY, Taslimi MM, Batzoglou S, Ronaghi M: Bacterial flora-typing with targeted, chip-based Pyrosequencing. BMC Microbiol 2007, 7:108.

28. Sanguinetti CJ, Dias-Neto E, Simpson AJ: Rapid silver staining and recovery of PCR products separated on polyacrylamide gels. Biotechniques 2004 17:914-921.

29. Caporaso JG, Kuczynski J, Stombaugh J, Bittinger K, Bushman FD, Costello EK, Fierer N, Peña AG, Goodrich JK, Gordon Jl, Huttley GA, Kelley ST, Knights D, Koenig JE, Ley RE, Lozupone CA, McDonald D, Muegge BD, Pirrung M, Reeder J, Sevinsky JR, Turnbaugh PJ, Walters WA, Widmann J, Yatsunenko T, Zaneveld J, Knight R: QIIME allows analysis of high-throughput community sequencing data. Nat Methods 2010, 7:335-336.

30. Edgar RC: UPARSE: Highly accurate OTU sequences from microbial amplicon reads. Nat Methods 2013, 10(10):996-998.

31. Edgar RC, Haas BJ, Clemente JC, Quince C, Knight R: UCHIME improves sensitivity and speed of chimera detection. Bioinformatics 2011. doi:10.1093/bioinformatics/btr38.

32. Altschul SF, Gish W, Miller W, Myers EW, Lipman DJ: Basic local alignment search tool. J Mol Biol 1990, 215:403-410. 
33. Quast C, Pruesse E, Yilmaz P, Gerken J, Schweer T, Yarza P, Peplies J, Glöckner FO: The SILVA ribosomal RNA gene database project: improved data processing and web-based tools. Nucleic Acids Res 2013, 41:D590-D596.

34. Sanapareddy N, Legge RM, Jovov B, McCoy A, Burcal L, Araujo-Perez F, Randall TA, Galanko J, Benson A, Sandler RS, Rawls JF, Abdo Z, Fodor AA, Keku TO: Increased rectal microbial richness is associated with the presence of colorectal adenomas in humans. ISME J 2012, 6:1858-1868.

35. Shannon CE, Weaver W: The mathematical theory of communication. AT\&T Tech J 1949, 27:359-423.

36. Chao A: Estimating the population size for capture-recapture data with unequal catchability. Biometrics 1987, 43:783-791.

37. Chao A, Lee SM: Estimating the number of classes via sample coverage. J Am Stat Assoc 1992, 87:210-217.

38. McMurdie PJ, Holmes S: phyloseq: An R package for reproducible interactive analysis and graphics of microbiome census data. PLoS One 2013, 8:e61217.

39. Morris A, Beck JM, Schloss PD, Campbell TB, Crothers K, Curtis JL, Flores SC, Fontenot AP, Ghedin E, Huang L, Jablonski K, Kleerup E, Lynch SV, Sodergren E, Twigg H, Young VB, Bassis CM, Venkataraman A, Schmidt TM, Weinstock GM: Comparison of the respiratory microbiome in healthy nonsmokers and smokers. Am J Respir Crit Care Med 2013, 187:1067-1075.

40. Ding T, Schloss PD: Dynamics and associations of microbial community types across the human body. Nature 2014, 509(7500):357-360.

41. Human Microbiome Project Consortium: Structure, function and diversity of the healthy human microbiome. Nature 2012,486:207-214.

42. Li K, Bihan M, Methé BA: Analyses of the stability and core taxonomic memberships of the human microbiome. PLoS One 2013, 6:8:e63139.

43. Charlson ES, Chen J, Custers-Allen R, Bittinger K, Li H, Sinha R, Hwang J, Bushman FD, Collman RG: Disordered microbial communities in the upper respiratory tract of cigarette smokers. PLoS One 2010, 5:e15216.

44. Bull-Otterson L, Feng W, Kirpich I, Wang Y, Qin X, Liu Y, Gobejishvili L, Joshi-Barve S, Ayvaz T, Petrosino J, Kong M, Barker D, McClain C, Barve S: Metagenomic Analyses of Alcohol Induced Pathogenic Alterations in the Intestinal Microbiome and the Effect of Lactobacillus rhamnosus GG Treatment. PLoS One 2013, 8:e53028.

45. Jabbour Z, do Nascimento C, Kotake BG, El-Hakim M, Henderson JE, de Albuquerque Junior RF: Assessing the oral microbiome of healthy and alcohol-treated rats using whole-genome DNA probes from human bacteria. Arch Oral Biol 2013, 58:317-323.

46. Walker AW, Sanderson JD, Churcher C, Parkes GC, Hudspith BN, Rayment N, Brostoff J, Parkhill J, Dougan G, Petrovska L: High-throughput clone library analysis of the mucosa-associated microbiota reveals dysbiosis and differences between inflamed and non-inflamed regions of the intestine in inflammatory bowel disease. BMC Microbiol 2011, 10:7.

47. Ismail IH, Oppedisano F, Joseph SJ, Boyle RJ, Licciardi PV, Robins-Browne $\mathrm{RM}$, Tang ML: Reduced gut microbial diversity in early life is associated with later development of eczema but not atopy in high-risk infants. Pediatr Allergy Immunol 2012, 23:674-681.

48. Manichanh C, Rigottier-Gois L, Bonnaud E, Gloux K, Pelletier E, Frangeul L, Nalin R, Jarrin C, Chardon P, Marteau P, Roca J, Dore J: Reduced diversity of faecal microbiota in Crohn's disease revealed by a metagenomic approach. Gut 2006, 55:205-211.

49. Le Chatelier E, Nielsen T, Qin J, Prifti E, Hildebrand F, Falony G, Almeida M, Arumugam M, Batto JM, Kennedy S, Leonard P, Li J, Burgdorf K, Grarup N, Jørgensen T, Brandslund I, Nielsen HB, Juncker AS, Bertalan M, Levenez F, Pons N, Rasmussen S, Sunagawa S, Tap J, Tims S, Zoetendal EG, Brunak S, Clément K, Doré J, Kleerebezem $M$, et al: Richness of human gut microbiome correlates with metabolic markers. Nature 2013 500(7464):541-546.

50. Chang JY, Antonopoulos DA, Kalra A, Tonelli A, Khalife WT, Schmidt TM, Young VB: Decreased diversity of the fecal Microbiome in recurrent Clostridium difficile-associated diarrhea. J Infect Dis 2008, 197:435-438.

51. Hajishengallis G, Darveau RP, Curtis MA: The keystone-pathogen hypothesis. Nature Reviews Microbiol 2012, 10:717-725.

52. Lee J, Taneja V, Vassallo R: Cigarette smoking and Inflammation: cellular and Molecular Mechanisms. J Dent Res 2012, 91:142-149.

53. Brinig MM, Lepp PW, Ouverney CC, Armitage GC, Relman DA: Prevalence of bacteria of division TM7 in human subgingival plaque and their association with disease. Appl Environ Microbiol 2003, 69:1687-1694.

54. Podar M, Abulencia CB, Walcher M, Hutchison D, Zengler K, Garcia JA, Holland T, Cotton D, Hauser L, Keller M: Targeted access to the genomes of low-abundance organisms in complex microbial communities. Appl Environ Microbiol 2007, 73:3205-3214.

55. Zaura E, Keijser BJ, Huse SM, Crielaard W: Defining the healthy "core microbiome" of oral microbial communities. BMC Microbio/ 2009, 9:259.

56. Liu Bl, Faller LL, Klitgord N, Mazumdar V, Ghodsi M, Sommer DD, Gibbons TR, Treangen TJ, Chang YC, Li S, Stine OC, Hasturk H, Kasif S, Segrè D, Pop M, Amar S: Deep sequencing of the oral microbiome reveals signatures of periodontal disease. PLoS One 2012, 7(6):e37919.

57. Benítez-Páez A, Belda-Ferre P, Simón-Soro A, Mira A: Microbiota diversity and gene expression dynamics in human oral biofilms. BMC Genomics 2014, 15:311.

58. Gong HL, Shi Y, Zhou L, Wu CP, Cao PY, Tao L, Xu C, Hou DS, Wang YZ: The Composition of Microbiome in Larynx and the Throat Biodiversity between Laryngeal Squamous Cell Carcinoma Patients and Control Population. PLoS One 2013, 8:e66476.

59. Charlson ES, Bittinger K, Haas AR, Fitzgerald AS, Frank I, Yadav A, Bushman FD Collman RG: Topographical continuity of bacterial populations in the healthy human respiratory tract. Am J Respir Crit Care Med 2011, 184:957-963.

60. Brook I: The role of bacterial interference in otitis, sinusitis and tonsillitis. Otolaryngol Head Neck Surg 2005, 133:139-146.

61. Darveau RP: Periodontitis: a polymicrobial disruption of host homeostasis. Nat Rev Microbiol 2010, 8:481-490.

62. Socransky S, Haffajee AD, Cugini MA, Smith C, Jr K: Microbial complexes in subgingival plaque. J Clin Periodontol 1998, 25(2):134-144.

63. Shchipkova AY, Nagaraja HN, Kumar PS: Subgingival Microbial Profiles of Smokers with Periodontitis. J Dent Res, 89:1247-1253.

64. Kembel SW, Wu M, Eisen JA, Green JL: Incorporating $16 \mathrm{~S}$ gene copy number information improves estimates of microbial diversity and abundance. PLoS Comput Biol 2012, 8:1002743.

65. Angly FE, Dennis PG, Skarshewski A, Vanwonterghem I, Hugenholtz P, Tyson GW: CopyRighter: a rapid tool for improving the accuracy of microbial community profiles through lineage-specific gene copy number correction. Microbiome 2014, 2:11.

66. Adler CJ, Dobney K, Weyrich LS, Kaidonis J, Walker AW, Haak W, Bradshaw CJ, Townsend G, Sołtysiak A, Alt KW, Parkhill J, Cooper A: Sequencing ancient calcified dental plaque shows changes in oral microbiota with dietary shifts of the Neolithic and Industrial revolutions. Nat Genetics 2013, 45:450-455.

doi:10.1186/s12866-014-0250-2

Cite this article as: Thomas et al:: Alcohol and tobacco consumption affects bacterial richness in oral cavity mucosa biofilms. BMC Microbiology 2014 14:250.

\section{Submit your next manuscript to BioMed Central and take full advantage of:}

- Convenient online submission

- Thorough peer review

- No space constraints or color figure charges

- Immediate publication on acceptance

- Inclusion in PubMed, CAS, Scopus and Google Scholar

- Research which is freely available for redistribution

Submit your manuscript at www.biomedcentral.com/submit
C) Biomed Central 